\title{
Habermas, Jürgen
}

\section{Die Idee der Universität. Lernprozesse}

Zeitschrift für Pädagogik 32 (1986) 5, S. 703-718

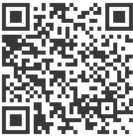

Quellenangabe/ Reference:

Habermas, Jürgen: Die Idee der Universität. Lernprozesse - In: Zeitschrift für Pädagogik 32

(1986) 5, S. 703-718 - URN: urn:nbn:de:0111-pedocs-144116 - DOI: 10.25656/01:14411

https://nbn-resolving.org/urn:nbn:de:0111-pedocs-144116

https://doi.org/10.25656/01:14411

in Kooperation mit / in cooperation with:

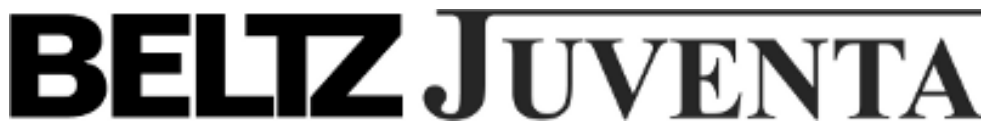

http://www.juventa.de

\section{Nutzungsbedingungen}

Gewährt wird ein nicht exklusives, nicht übertragbares, persönliches und beschränktes Recht auf Nutzung dieses Dokuments. Dieses Dokument ist ausschließlich für den persönlichen, nicht-kommerziellen Gebrauch bestimmt. Die Nutzung stellt keine Übertragung des Eigentumsrechts an diesem Dokument dar und gilt vorbehaltlich der folgenden Einschränkungen: Auf sämtlichen Kopien dieses Dokuments müssen alle Urheberrechtshinweise und sonstigen Hinweise auf gesetzlichen Schutz beibehalten werden. Sie dürfen dieses Dokument nicht in irgendeiner Weise abändern, noch dürfen Sie dieses Dokument für öffentliche oder kommerzielle Zwecke vervielfältigen, öffentlich ausstellen, aufführen, vertreiben oder anderweitig nutzen.

Mit der Verwendung dieses Dokuments erkennen Sie die Nutzungsbedingungen an.

\section{Terms of use}

We grant a non-exclusive, non-transferable, individual and limited right to using this document.

This document is solely intended for your personal, non-commercial use. Use of this document does not include any transfer of property rights and it is conditional to the following limitations: All of the copies of this documents must retain all copyright information and other information regarding legal protection. You are not allowed to alter this document in any way, to copy it for public or commercial purposes, to exhibit the document in public, to perform, distribute or otherwise use the document in public.

By using this particular document, you accept the above-stated conditions of use.

\section{Kontakt / Contact:}

peDOcs

DIPF | Leibniz-Institut für Bildungsforschung und Bildungsinformation Informationszentrum (IZ) Bildung

E-Mail: pedocs@dipf.de

Internet: www.pedocs.de

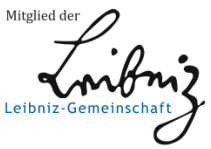




\section{Zeitschrift für Pädagogik}

\section{Jahrgang 32 - Heft 5 - Oktober 1986}

\section{Essay}

ANDREAS FLITNER
Erasmus von Rotterdam - Lehrer der Humanitas, Lehrer des Friedens 605

II. Thema: Empirische Schul- und Unterrichtsforschung

Hans Aebli/

URSULA RuthEMANN/

Fritz StAub

JÜRgen BAUMERT/

Peter Martin Roeder/

FRITZ SANG/

BERND SCHMITZ

ANNE SCHMIDT/

JÜRGEN VAN BUER/

BIRGIT REISING

JÜRGEN VAN BUER/

Frank ACHTENHagen/

Hartmut Oldenbürger
Sind Regeln des Problemlösens lehrbar? 617

Leistungsentwicklung und Ausgleich von Leistungsunterschieden in Gymnasialklassen 639

Zur impliziten Persönlichkeitstheorie von Lehrern an beruflichen Schulen im allgemein-gewerblichen Bereich. Untersuchungen zu Unterschieden zwischen den Lehrern 661

Lehrerurteile über Schüler, Schülerselbstbild und interaktionelles Verhalten im Englischanfangsunterricht 679

\section{Weitere Beiträge}

JÜRgen HABERMAS

Die Idee der Universität - Lernprozesse 703

OTTO FRIEDRICH

BOLLNOW

Einige Bemerkungen zu Schleiermachers Pädagogik 719

Heinz-Elmar Tenorth (Hrsg.): Allgemeine Bildung. Analysen zu ihrer Wirklichkeit, Versuche über ihre Zukunft 743 
FRIEDHELM BRÜGGEN GüNTHER Buck: Rückwege aus der Entfremdung. Studien zur Entwicklung der deutschen humanistischen Bildungsphilosophie 747

Heinz-Elmar Tenorth Manfred Eckert: Die schulpolitische Instrumentalisierung des Bildungsbegriffs. Zum Abgrenzungsstreit zwischen Realschule und Gymnasium im 19. Jahrhundert 753

JÜRGEN DIEDERICH

Barbara GaEbe: Lehrplan im Wandel. Veränderungen in den Auffassungen und Begründungen von Schulwissen 755

\section{Dokumentation}

Pädagogische Neuerscheinungen 761 


\section{Contents}

\section{Essay}

ANDREAS FlitNer

II. Topic

Hans AEBLI/

URSULA RUTHEMANN/

Fritz StAub

JÜRgen BAUMERT/

Peter Martin Roeder/

FRITZ SANG/

BERND SCHMITZ

ANNE SCHMIDT/

JÜRGEN VAN BUER/

Birgit REISING

JÜRGEN VAN BUER/

Frank Achtenhagen/

HARTMUT OldenbÜRGER
Erasmus - Teacher of Humanitas and Peace 605

Are Rules of Problem Solving teachable? 617
Achievement Growth and the Reduction of Achievement Differentials within Classrooms 639

On the Implicit Theory of Personality Entertained by Teachers in Vocational Schools 661

Teachers' Assessment of Pupils, Pupils' Self-Concept and Interactional Behaviour in Initial English Courses 679

\section{Other Contributions}

JÜRgEN HABERMas

The Concept of University - Learning Processes

703

OTTO FRIEDRICH

On Schleiermacher's Educational Theory 719

BOLLNOW

IV. Book Reviews

743

V. Documentation

New Books 769 


\section{Die Idee der Universität - Lernprozesse*}

\section{Zusammenfassung}

Der Aufsatz geht aus von der Idee der Universität, die die preußischen Hodhschulreformer Anfang des 19. Jahrhunderts entworfen haben. Er analysiert, auf welche Weise die Reformschriften der sechziger Jahre - JASPERs, SCHELSKY und die SDS-Denkschrift - jeweils an diese Tradition anknüpfen und die klassische Universitätsidee umformen. Dabei geht es um die Frage, ob die funktionsbündelnde Institution der Hochschule auch heute noch auf ein integrierendes Selbstverständnis angewiesen ist - oder ob die Universität als Teil eines ausdifferenzierten Wissenschaftssystems auch ganz gut ohne eine Idee von selber funktionieren kann.

\section{Einleitung: Die Idee der Universität und das moderne Hochschulsystem}

Im ersten Heft des ersten Jahrgangs der damals von KARL JASPERS und ALFRED Weber, Dolf Sternberger und AleXANDer Mitscherlich gegründeten Zeitschrift „Die Wandlung“ kann man die Rede nachlesen, die der aus der inneren Emigration auf seinen Lehrstuhl zurückkehrende Philosoph im Jahre 1945 zur Wiedereröffnung der Universität Heidelberg gehalten hat: KARL JASPERS „Die Erneuerung der Universität". Mit der Emphase des neuen Anfangs, den die zeitgeschichtliche Situation in Aussicht stellte, griff JASPERS damals den zentralen Gedanken aus seiner Schrift über die „Idee der Universität“ auf, die zuerst 1923 erschienen war und 1946 neu aufgelegt wurde. Fünfzehn Jahre später erscheint das Buch in neuer Bearbeitung (JASPERS/RosSMANN 1961). Immer noch geht JASPERS unbefangen von Prämissen aus, die sich der impliziten Soziologie des Deutschen Idealismus verdanken: Eine Institution bleibt nur solange funktionsfähig, wie sie die ihr innewohnende Idee lebendig verkörpert. Sobald der Geist aus ihr entweicht, erstarrt eine Institution in ähnlicher Weise zu etwas bloß Mechanischem, wie sich der seelenlose Organismus in tote Materie auflöst.

Auch die Universität bildet kein Ganzes mehr, sobald das einigende Band ihres korporativen Bewußtseins zerfällt. Die Funktionen, die die Universität für die Gesellschaft erfüllt, müssen gleichsam von innen mit den Zielsetzungen, Motiven und Handlungen ihrer arbeitsteilig kooperierenden Mitglieder vereinigt bleiben. In diesem Sinne soll die Universität eine von ihren Angehörigen intersubjektiv geteilte, sogar eine exemplarische Lebensform institutionell verkörpern und zugleich motivational verankern. Was seit HuMBoLDT die „Idee der Universität“ heißt, ist das Projekt der Verkörperung einer idealen Lebensform. Diese Idee soll sich vor anderen Gründungsideen noch dadurch auszeichnen, daß sie nicht nur auf eine der vielen partikularen Lebensformen der frühbürgerlichen, berufsständisch stratifizierten Gesellschaft verweist, sondern - dank ihrer Verschwisterung mit Wissenschaft und Wahrheit - auf ein Allgemeines, dem Pluralismus gesellschaftlicher Lebensformen Vorgängiges. Die Idee der Universität verweist auf die Bildungsgesetze, nach denen sich alle Gestalten des objektiven Geistes formieren. 
Selbst wenn wir von diesem überschwenglichen Anspruch aufs Exemplarische absehen - ist nicht schon die Prämisse, daß ein unübersichtliches Gebilde wie das moderne Hochschulsystem von der gemeinsamen Denkungsart ihrer Mitglieder durchdrungen und getragen werden müsse, unrealistisch? Hätte JASPERS nicht schon von MAX WEBER gelernt haben können, daß die organisationsförmige Realität, in der sich die funktional spezifizierten Teilsysteme einer hoch differenzierten Gesellschaft sedimentieren, auf ganz anderen Prämissen beruht? Die Funktionsfähigkeit solcher Betriebe und Anstalten hängt gerade davon $a b$, daß die Motive der Mitglieder von den Organisationszielen und -funktionen entkoppelt sind. Organisationen verkörpern keine Ideen mehr. Wer sie auf Ideen verpflichten wollte, müßte ihren operativen Spielraum auf den vergleichsweise engen Horizont der von den Mitgliedern intersubjektiv geteilten Lebenswelt beschränken. Einer der vielen huldigenden Artikel, mit denen die FAZ die Universität Heidelberg zu ihrem 600. Geburtstag verwöhnt, kommt denn auch zu dem ernüchternden Schluß: „Das Bekenntnis zu HuMBOLDT ist die Lebenslüge unserer Universitäten. Sie haben keine Idee" (REUMANN 1986). Aus dieser Sicht gehören alle jene Universitätsreformer, die sich wie JASPERS auf die Idee der Universität berufen haben und noch berufen, zu den bloß defensiven Geistern einer modernisierungsfeindlichen Kulturkritik.

Nun war JASPERS von den idealistischen Zügen eines soziologiefremden, bildungselitären, bürgerlichen Kulturpessimismus, d.h. von der Hintergrundideologie der deutschen Mandarine gewiß nicht frei; aber er war nicht der einzige, nicht einmal der einflußreichste unter denen, die in den sechziger Jahren eine überfällige Universitätsreform mit Rückgriff auf die Ideen der preußischen Universitätsreformer eingeklagt haben. Im Jahre 1963, zwei Jahre nach der Neufassung der Jasperschen Schrift, hat sich Helmut SCHELSKY mit seinem schon im Titel unverkennbaren Buch „Einsamkeit und Freiheit“ zu Wort gemeldet. Und wiederum zwei Jahre später erschien die Ausarbeitung einer (zunächst 1961 vorgestellten) Denkschrift des SDS unter dem Titel „Hochschule in der Demokratie“ (NITSCH u. a. 1965). Drei Reformschriften aus drei Generationen und drei verschiedenen Perspektiven. Sie markieren einen jeweils größer gewordenen Abstand von HuMBOLDT - und eine wachsende sozialwissenschaftliche Ernüchterung über die Idee der Universität. Trotz des Generationenabstandes und des eingetretenen Mentalitätswandels ließ sich freilich keine dieser drei Parteien ganz davon abbringen, daß es um eine kritische Erneuerung eben jener Idee gehe.

Zwanzig Jahre und eine halbherzig durchgeführte, teils wieder zurückgenommene Organisationsreform der Hochschule trennen uns heute von diesen Versuchen, der Universität im Lichte ihrer erneuerten Idee eine neue Gestalt zu geben. Es sieht so aus, als behielten jene Realisten recht, die, wie JASPERS anmerkt, schon nach dem Ersten Weltkrieg erklärt hatten: „Die Idee der Universität ist tot! Lassen wir die Illusion fallen! Jagen wir nicht Fiktionen nach!" (JASPERS 1961, S. 7). Oder hatten wir nur die Rolle, die eine solche Idee für das Selbstverständnis universitär organisierter Lernprozesse nach wie vor spielen könnte, nicht richtig verstanden? Mußte die Universität auf ihrem Wege zur funktionalen Spezifizierung innerhalb eines beschleunigt ausdifferenzierten Wissenschaftssystems das, was man einmal ihre Idee genannt hat, als leere Hülse abstreifen? Oder ist die universitäre Form organisierter wissenschaftlicher Lernprozesse auch heute noch auf eine Bündelung 
von Funktionen angewiesen, die nicht gerade ein normatives Leitbild erfordert, aber doch eine gewisse Gemeinsamkeit in den Selbstdeutungen der Universitätsangehörigen - Reste eines korporativen Bewußtseins?

\section{Das moderne Hochschulsystem und seine funktionalistische Deutung}

Vielleicht genügt schon ein Blick auf die äußere Entwicklung der Hochschulen, um diese Fragen zu beantworten. Die Bildungsexpansion nach dem zweiten Weltkrieg ist ein weltweites Phänomen, das T. PARsons veranlaßt hat, von einer Erziehungsrevolution zu sprechen.

Im Deutschen Reich war die Zahl der Studierenden zwischen 1933 und 1939 von 121000 auf 56000 Studenten halbiert worden. 1945 bestanden auf dem Gebiet der späteren Bundesrepublik nur noch 15 Hochschulen. Mitte der fünfziger Jahre versorgten 50 wissenschaftliche Hochschulen schon wieder etwa 150000 Studenten. Anfang der sechziger Jahre wurden dann die Weichen für einen gezielten Ausbau des tertiären Sektors gestelit. Seitdem hat sich die Studentenzahl noch einmal vervierfacht. Heute werden über eine Million Studenten an 94 wissenschaftlichen Hochschulen ausgebildet ${ }^{1}$. Diese absoluten Zahlen gewinnen freilich einen informativen Gehalt erst im internationalen Vergleich. In fast allen westlichen Industriegesellschaften beginnt nach 1945 der Trend zur Erweiterung der formalen Bildung und setzt sich verstärkt bis Ende der siebziger Jahre fort; in den entwickelten sozialistischen Gesellschaften konzentriert sich der gleiche Erweiterungsschub in den fünfziger Jahren. In allen Industrieländern nahm die von der UNESCO für den Zeitraum von 1950 bis 1980 brutto berechnete Sekundarschulrate von 30 auf 80, die entsprechende Hochschulrate von knapp 4 auf $30 \mathrm{zu}$. Die Parallelen in der Bildungsexpansion verschiedener Industriegesellschaften zeigen sich noch deutlicher, wenn man, wie im angekündigten zweiten Bildungsbericht des MPI für Bildungsforschung, die Selektivität des Bildungssystems in der Bundesrepublik mit dem in den USA, Großbritannien, Frankreich und der DDR vergleicht. Obwohl die nationalen Bildungssysteme ganz verschieden aufgebaut sind, und trotz der Unterschiede im politischen und gesellschaftlichen System, ergeben sich für die höchsten Qualifikationsstufen dieselben Größenordnungen. Wenn man die Bildungselite an höheren akademischen Graden (in der Regel an der Promotion) mißt, liegt ihr Anteil zwischen 1,5\% und 2,6\% eines Jahrgangs; mißt man sie am Abschluß der wichtigsten Formen des akademischen Langzeitstudiums (an Diplom, Magister und Staatsexamen), liegt der Anteil zwischen 8\% und 10\% eines Jahrgangs. Die Autoren des zweiten Bildungsberichts vertiefen diesen Vergleich bis in Bereiche der qualitativen Differenzierung und stellen beispielsweise fest, daß sich das Publikationsverhalten und die nach äußeren Indikatoren eingeschätzte wissenschaftliche Produktivität in den einzelnen Fächern international auf verblüffende Weise ähneln - ganz unabhängig davon, ob die nationalen Hochschulsysteme eher offen strukturiert oder stärker auf Auswahl und Elitenbildung zugeschnitten sind. Die deutschen Universitäten haben sich zudem, bei aller steifnackigen Resistenz gegen die staatlich verordnete Reform, nicht nur in den quantitativen Dimensionen verändert. Mit der Ordinarienuniversität sind überholte Hierarchien abgebaut worden; mit einer gewissen Statusnivellierung verlor auch die Mandarinenideologie ihre Grundlage. Auslagerungen und interne Differenzierungen haben Lehre und Forschung stärker auseinandertreten lassen. Mit einem Wort: auch in ihren inneren Strukturen haben sich in der Bundesrepublik die Massenuniversitäten den Hochschulen anderer Industrieländer angeglichen.

Aus der Entfernung des international vergleichenden bildungssoziologischen Blicks bietet sich also ein Bild, das eine funktionalistische Deutung aufdrängt. Danach bestimmen die Gesetzmäßigkeiten der gesellschaftlichen Modernisierung auch die 
Hochschulentwicklung, die freilich in der Bundesrepublik, im Vergleich zur DDR und zum westlichen Ausland, mit einem Jahrzehnt Verspätung eingesetzt hat. In der Phase größter Beschleunigung hat dann die Bildungsexpansion entsprechende Ideologien erzeugt. Der Streit zwischen den Reformern und den Verteidigern eines unhaltbar gewordenen Status quo ist damals von allen Seiten, so scheint es, unter der falschen Prämisse ausgetragen worden, daß es darum gehe, die Idee der Universität sei es zu erneuern oder zu bewahren. In diesen ideologischen Formen hat sich ein Proze $\beta$ vollzogen, den keine der beiden Parteien gewollt hat - und den die revoltierenden Studenten seinerzeit als "technokratische Hochschulreform" bekämpft hatten. Im Zeichen der Reform scheint sich bei uns nur ein neuer Schub in der Ausdifferenzierung eines Wissenschaftssystems vollzogen zu haben, das sich wie überall funktional verselbständigt hat - und das normativer Integration in den Köpfen von Professoren und Studenten umso weniger bedarf, je mehr es sich über systemische Mechanismen steuert und sich mit der disziplinären Erzeugung von technisch verwertbaren Informationen und Berufsqualifikationen auf die Umwelten der Ökonomie und der planenden Verwaltung einstellt. Zu diesem Bild passen die pragmatischen Empfehlungen des Wissenschaftsrates, die eine Verlagerung der Gewichte zugunsten systemischer Steuerung, zugunsten disziplinärer Eigenständigkeit, zugunsten einer Differenzierung von Forschung und Lehre fordern (s. WISSENSCHAFTSRAT $1984 ; 1985 \mathrm{a}, \mathrm{b} ; 1986)$.

Freilich läßt die ideenpolitische Enthaltsamkeit des Wissenschaftsrats weitergreifende Interpretationen offen. Die vorsichtigen Empfehlungen implizieren nicht notwendig jene funktionalistische Lesart, die sich mit einem heute verbreiteten neokonservativen Deutungsmuster trifft. Einerseits setzt man aufs funktional ausdifferenzierte Wissenschaftssystem, für das die normativ integrierende Kraft eines im korporativen Selbstverständnis verankerten ideellen Zentrums nur hinderlich wäre; andererseits nimmt man die Jubiläumsdaten gerne zum rhetorischen Anlaß, um über einer systemisch geronnenen Autonomie der Hochschule den Traditionsmantel einer einst ganz anders, nämlich normativ gemeinten Autonomie auszubreiten. Unter diesem Schleier können sich dann die Informationsflüsse zwischen den funktional autonom gewordenen Teilsystemen, zwischen den Hochschulen und dem ökonomisch-militärisch-administrativen Komplex umso unauffälliger einspielen. Aus dieser Sicht wird das Traditionsbewußtsein nur noch an seinem Kompensationswert gemessen; es ist soviel wert wie die Löcher groß sind, die es in einer ihrer Idee beraubten Universität zu stopfen vermag. Auch diese neokonservative Deutung könnte freilich, soziologisch betrachtet, der bloße Reflex einer Bildungskonjunktur sein, die ihren Zyklen folgt - ganz unbeirrt von den Politiken, den Themen und den Theorien, denen sie jeweils zum Aufschwung verhilft.

Die aktive Bildungspolitik, die bei uns im Zuge eines überfälligen Modernisierungsschubes Anfang der sechziger Jahre eingesetzt hatte, beruhte bis zum Ende der Großen Koalition auf einem tragfähigen Konsens aller Parteien; während der ersten Regierung BRANDT hat die Bundesrepublik dann eine bildungspolitische Hochkonjunktur erlebt - und den Beginn der Polarisierung. Seit 1974 setzte schließlich der Abschwung ein, weil seitdem die Bildungspolitik durch die einsetzende Wirtschaftskrise von beiden Seiten betroffen wurde: auf der Absolventenseite durch erschwerte Arbeitsmarktbedingungen, auf der Kosten- und Finanzierungsseite durch die Krise der öffentlichen Haushalte (HúfNer u. a. 1986, S. $200 \mathrm{ff}$.; vgl. auch HÜNER u. a. 1977). Man kann also das, was den Neokonservativen heute als realistische 
Neuorientierung der Bildungspolitik erscheint, auch als ein weitgehend ökonomisch und politisch zu erklärendes Rezessionsphänomen der Bildungsplanung verstehen ${ }^{2}$.

Wenn aber die Bildungskonjunkturen gleichsam durch die Themen und Theorien hindurchgreifen, ist auch die funktionalistische Deutung, die heute dominiert, nicht schlicht at face value zu nehmen. Prozesse der Ausdifferenzierung, die sich in den beiden letzten Jahrzehnten beschleunigt haben, müssen nicht unter eine systemtheoretische Beschreibung gebracht werden und $\mathrm{zu}$ dem Schluß führen, daß die Universitäten den lebensweltlichen Horizonten nun ganz entwachsen sind.

Empirisch gesehen, scheint es eine offene Frage zu sein, ob nicht die Antriebe wissenschaftlicher Lernprozesse am Ende erlahmen müßten, wenn diese ausschließlich auf die Funktion der Forschung spezialisiert wären. Wissenschaftliche Produktivität könnte sehr wohl von universitären Formen der Organisation abhängig sein, nämlich angewiesen auf jenen in sich differenzierten Komplex der Förderung des wissenschaftlichen Nachwuchses, der Vorbereitung auf akademische Berufe sowie der Beteiligung an Prozessen der Allgemeinbildung, der kulturellen Selbstverständigung und der öffentlichen Meinungsbildung. Noch sind die Hochschulen über diese merkwürdige Bündelung von Funktionen in der Lebenswelt verwurzelt. Solange dieser Zusammenhang nicht vollends reißt, kann die Idee der Universität immerhin nicht ganz tot sein. Die Komplexität und innere Differenzierung dieses Zusammenhangs darf freilich nicht unterschätzt werden. In der Geburtsstunde der klassischen deutschen Universität haben die preußischen Reformer ein Bild von ihr entworfen, das einen übervereinfachten Zusammenhang zwischen wissenschaftlichen Lernprozessen und den Lebensformen moderner Gesellschaften suggeriert.

\section{Die klassische Idee der Universität: SCHELLING, HUMBOLDT, SCHLEIERMACHER}

Humboldt und SCHLEIERMACHER verknüpfen mit der Idee der Universität zwei Gedanken. Erstens geht es ihnen um das Problem, wie die moderne, aus der Vormundschaft von Religion und Kirche entlassene Wissenschaft institutionalisiert werden kann, ohne daß ihre Autonomie von anderer Seite gefährdet wird - sei es durch die Befehle der staatlichen Obrigkeit, die die äußere Existenz der Wissenschaft ermöglicht, oder durch Einflüsse der bürgerlichen Gesellschaft, die an den nützlichen Resultaten der wissenschaftlichen Arbeit interessiert ist. HUMBOLDT und SCHLEIERMACHER sehen die Lösung des Problems in einer staatlich organisierten Wissenschaftsautonomie, die die höheren wissenschaftlichen Anstalten gegen politische Eingriffe ebenso wie gegen gesellschaftliche Imperative abschirmt. Zum anderen wollen HUMBOLDT und SCHLEIERMACHER auch erklären, warum es im Interesse des Staates selber liegt, der Universität die äußere Gestalt einer nach innen unbeschränkten Freiheit zu garantieren. Wenn nur die wissenschaftliche Arbeit der inneren Dynamik der Forschungsprozesse überlassen würde (s. HuMBoLdT [1810] 1959, S. 379), dann müßte sich, davon waren beide überzeugt, die moralische Kultur, überhaupt das geistige Leben der Nation in den höheren wissenschaftlichen Anstalten wie in einem Focus zusammenfassen ${ }^{3}$.

Diese beiden Gedanken verschmelzen zur Idee der Universität und erklären einige der auffälligeren Eigenschaften der deutschen Universitätstradition. Sie machen erstens das affirmative Verhältnis einer sich unpolitisch verstehenden Universitäts- 
wissenschaft zum Staat verständlich, zweitens das defensive Verhältnis der Universität zur beruflichen Praxis, insbesondere zu Ausbildungsanforderungen, die das Prinzip der Einheit von Lehre und Forschung gefährden könnten, und drittens die zentrale Stellung der philosophischen Fakultät innerhalb der Hochschule sowie die emphatische Bedeutung, die der Wissenschaft für Kultur und Gesellschaft im ganzen zugeschrieben wird. Aus der Universitätsidee ergibt sich also einerseits die entwicklungsträchtige, weil auf die funktionale Eigenständigkeit des Wissenschaftssystems verweisende Betonung der Wissenschaftsautonomie, welche freilich nur in „Einsamkeit und Freiheit“, aus der Distanz zur bürgerlichen Gesellschaft und zur politischen Öffentlichkeit wahrgenommen werden sollte, und andererseits die allgemein kulturprägende Kraft einer Wissenschaft, in der sich die Totalität der Lebenswelt reflexiv zusammenfassen sollte.

Die Reformer konnten sich damals den Wissenschaftsproze $ß$ als einen narzißtisch in sich geschlossenen Kreisprozeß forschenden Lehrens vorstellen, weil die Philosophie des Deutschen Idealismus von sich aus die Einheit von Lehre und Forschung erforderte. Während heute eine Diskussion auf dem jeweils neuesten Stand der Forschung und die Darstellung dieses Wissensstandes für Zwecke des Studiums zwei verschiedene Dinge sind, hatte SCHELLING in seinen „Vorlesungen zur Methode des akademischen Studiums" gezeigt, daß aus der Konstruktion des philosophischen Gedankens selber die Form seiner pädagogischen Vermittlung hervorgeht (SCHELLING [1802] 1959, S. 20). Dieser Typus von Theorie erforderte einen konstruktiven Aufbau, der mit dem Curriculum ihrer Darstellung zusammenfiel.

Auf die gleiche Weise sollte die Universität ihren inneren Bezug zur Lebenswelt der totalisierenden Kraft der Wissenschaft verdanken können. Der Philosophie trauten die Reformer eine einheitsstiftende Kraft unter drei Aspekten zu - nämlich im Hinblick, wie wir heute sagen würden, auf kulturelle Überlieferung, auf Sozialisation und auf gesellschaftliche Integration. Die philosophische Grundwissenschaft war erstens enzyklopädisch angelegt und konnte als solche sowohl die Einheit in der Mannigfaltigkeit der wissenschaftlichen Disziplinen sichern wie auch die Einheit der Wissenschaft mit Kunst und Kritik auf der einen, Recht und Moral auf der anderen Seite. Die Philosophie empfahl sich als Reflexionsform der Kultur im ganzen. Ihr platonistischer Grundzug sollte zweitens die Einheit von Forschungsprozessen und Bildungsprozessen sichern. Indem nämlich Ideen erfaßt werden, bilden sie sich zugleich in den sittlichen Charakter des Erkennenden ein und befreien diesen von aller Einseitigkeit. Die Erhebung zum Absoluten öffnet den Weg zur allseitigen Entfaltung der Individualität. Weil der Umgang mit dieser Art von Wissenschaft vernünftig macht, können „die Pflanzschulen der Wissenschaft zugleich allgemeine Bildungsanstalten" sein (SCHELLING [1802] 1959, S. 21). Schließlich versprach die reflexionsphilosophische Grundlage aller Theoriebildung die Einheit von Wissenschaft und Aufklärung. Während heute die Philosophie ein Fach geworden ist, das das esoterische Interesse von Fachleuten auf sich zieht, konnte eine Philosophie, die von der Selbstbeziehung des erkennenden Subjekts ausging und alle Erkenntnisinhalte auf dem Weg einer reflexiven Denkbewegung entfaltete, das esoterische Interesse des Fachmanns an der Wissenschaft gleichzeitig mit dem exoterischen Interesse des Laien an Selbstverständigung und Aufklärung befriedigen (MARTENs/ SCHNÄDElBaCh 1985, S. $22 \mathrm{ff}$.). Indem die Philosophie, wie Hegel sagen wird, ihre Zeit in Gedanken erfaßt, sollte sie die sozial-integrative Kraft der Religion 
durch die versöhnende der Vernunft ersetzen. Deshalb konnte FICHTE die Universität, die eine solche Wissenschaft bloß institutionalisiert, als Geburtsstätte einer künftigen, emanzipierten Gesellschaft verstehen, sogar als Stätte der nationalen Erziehung (FICHTE 1959, S. 217).

Das Riskante und Unwahrscheinliche jener Universitätsidee wird erst in ganzem Umfange deutlich, wenn man sich die Bedingungen klar macht, die für die Institutionalisierung einer solchen Wissenschaft hätten erfüllt sein müssen - einer Wissenschaft also, die allein durch ihre innere Struktur die Einheit von Forschung und Lehre, die Einheit der Wissenschaften, die Einheit von Wissenschaft und allgemeiner Bildung sowie die Einheit von Wissenschaft und Aufklärung zugleich ermöglichen und garantieren sollte.

Die strikt verstandene Einheit von Forschung und Lehre bedeutet, daß nur so gelehrt und gelernt wird, wie es für den innovativen Prozeß des wissenschaftlichen Fortschrittes nötig ist. Die Wissenschaft soll sich auch in dem Sinne selbst reproduzieren können, daß die Professoren ihren eigenen Nachwuchs heranbilden. Der künftige Forscher ist das einzige Ziel, für das die Universität der forschenden Gelehrten Ausbildungsaufgaben übernimmt. Immerhin behielt diese Beschränkung der akademischen Berufsvorbereitung auf die Förderung des wissenschaftlichen Nachwuchses wenigstens für die philosophische Fakultät eine gewisse Plausibilität, solange sich die Professorenschaft aus dem Kreis der von ihnen ausgebildeten Gymnasiallehrer ergänzte. Weiterhin konnte die Idee der Einheit der Wissenschaften nur geltend gemacht werden, wenn sich die oberen Fakultäten der wissenschaftlichen Führung einer völlig umgewandelten Artistenfakultät unterordneten und wenn die Philosophie, die hier ihren Sitz hatte, tatsächlich zur Grundwissenschaft der vereinigten Natur- und Geisteswissenschaften avancierte. Das ist der Sinn der Polemik gegen die Brotwissenschaften, gegen die Zerstreuung in Spezialschulen, gegen das bloß Abgeleitete jener Fakultäten, „die ihre Einheit nicht in der Erkenntnis unmittelbar, sondern in einem äußeren Geschäfte" finden. Als zwingende, aber von Anbeginn kontrafaktisch erhobene Konsequenz ergab sich die Forderung nach der Herrschaft der philosophischen Fakultät, ,weil alle Mitglieder der Universität, zu welcher Fakultät sie auch gehören, in ihr müssen eingewurzelt sein“" (SCHLEIERMACHER [1808] 1959, S. 259 ff.).

Die Einheit von Wissenschaft und allgemeiner Bildung hatte institutionell die Einheit der Lehrenden und Lernenden zur Voraussetzung (s. HuMBoldT [1810] 1959, S. 378). Dieses auf Kooperation angelegte, grundsätzlich egalitäre Ergänzungsverhältnis sollte in den diskursiven Formen des Seminarbetriebs verwirklicht werden. Es war unvereinbar mit der Personalstruktur, die sich schon bald in den hierarchisch gegliederten Instituten einer am Vorbild der experimentellen Naturwissenschaften orientierten Forschung herausbildete.

Überschwenglich war schließlich die Idee der Einheit von Wissenschaft und Aufklärung, soweit sie die Autonomie der Wissenschaften mit der Erwartung befrachtete, daß die Universität innerhalb ihrer Mauern wie in einem Mikrokosmos eine Gesellschaft von Freien und Gleichen antizipieren könne. Die philosophische Wissenschaft schien derart die allgemeinen Kompetenzen der Gattung in sich zusammenzufassen, daß die höheren wissenschaftlichen Anstalten für HUMBOLDT nicht nur als Spitze des gesamten Bildungssystems galten, sondern als „Gipfel der 
moralischen Kultur der Nation". Freilich blieb von Anfang an unklar, wie der aufklärerisch-emanzipatorische Auftrag mit der politischen Enthaltsamkeit zusammengehen sollte, die doch die Universität als Preis für die staatliche Organisation ihrer Freiheit entrichten mußte.

Diese institutionellen Voraussetzungen für eine Implementierung der Gründungsidee der deutschen Universität waren entweder von Anfang an nicht gegeben oder konnten im Laufe des 19. Jahrhunderts immer weniger erfüllt werden. Ein differenziertes Beschäftigungssystem erforderte erstens die wissenschaftliche Vorbereitung auf immer mehr akademische Berufe. Die Technischen Hochschulen, Handelshochschulen, Pädagogische Hochschulen, Kunsthochschulen konnten nicht auf Dauer neben den Universitäten bestehen bleiben. Sodann folgten die aus dem Schoß der philosophischen Fakultät entspringenden Erfahrungswissenschaften einem methodischen Ideal der Verfahrensrationalität, das jeden Versuch der enzyklopädischen Einbettung ihrer Inhalte in eine philosophische Gesamtdeutung zum Scheitern verurteilte. ${ }^{5}$ Diese Emanzipation der Erfahrungswissenschaften besiegelte den Zerfall einheitlich-metaphysischer Weltdeutungen. Inmitten eines Pluralismus von Glaubensmächten verlor die Philosophie auch ihr Monopol für die Deutung der Kultur im ganzen. Drittens avancierte die Wissenschaft zu einer wichtigen Produktivkraft der industriellen Gesellschaft. Die Naturwissenschaften büßten ihre Weltbildfunktion zugunsten der Erzeugung technisch verwertbaren Wissens ein. Die Arbeitsbedingungen der institutsförmig organisierten Forschung waren weniger auf Funktionen allgemeiner Bildung als auf die funktionalen Imperative von Wirtschaft und Verwaltung zugeschnitten. Schließlich diente die akademische Bildung in Deutschland der sozialen Abgrenzung einer am Modell des höheren Beamten orientierten bildungsbürgerlichen Schicht (FRIEDEBURG 1986, S. $23 \mathrm{ff}$.). Mit dieser Befestigung der berufsständischen Differenzierung zwischen Volksbildung und akademischer Bildung wurden aber Klassenstrukturen bestätigt, die den universalistischen Gehalt der Universitätsidee und das Versprechen, das diese für die Emanzipation der Gesellschaft im ganzen verheißen hatte, nachhaltig dementieren (s. ELLWEIN 1985, S. 124 ff.).

Je stärker diese gegenläufigen Entwicklungen zu Bewußtsein kamen, um so mehr mußte die Idee der Universität gegen die Tatsachen behauptet werden - sie verkam zur Ideologie eines Berufsstandes mit hohem sozialen Prestige. Für die Geistes- und Sozialwissenschaften datiert FRITZ K. RINGER (1969) den Verfall der Kultur der deutschen Mandarine auf die Periode von 1890 bis 1933. In der machtgeschützten Innerlichkeit dieser Mandarine hat sich das neuhumanistische Bildungsideal zu dem geistesaristokratischen, unpolitischen, obrigkeitskonformen Selbstverständnis einer praxisfernen, nach innen autonomen, forschungsintensiven Bildungsanstalt verformt ${ }^{6}$. Man muß freilich auch die positive Seite sehen. Die Idee der Universität hat in beiderlei Gestalt - sowohl als Idee wie als Ideologie - zu dem Glanz und dem international unvergleichlichen Erfolg der deutschen Universitätswissenschaft im 19. Jahrhundert, sogar bis in die dreißiger Jahre unseres Jahrhunderts hinein, beigetragen. Sie hat nämlich mit der staatlich organisierten Wissenschaftsautonomie die Ausdifferenzierung der wissenschaftlichen Disziplinen der freigesetzten inneren Dynamik der Forschungsprozesse selbst überantwortet. Unter dem Schirm eines nur äußerlich rezipierten Bildungshumanismus haben die Naturwissenschaften alsbald ihre Autonomie gewonnen und sind mit ihrer institutsförmig organisierten 
Forschungsarbeit auch für die zunächst seminaristisch betriebenen Geistes- und Sozialwissenschaften zu einem, bei allem Positivismus fruchtbaren Vorbild geworden $^{7}$. Gleichzeitig hat die Ideologie der deutschen Mandarine der Hochschule ein starkes korporatives Selbstbewußtsein, Förderung von seiten des Kulturstaates und eine gesamtgesellschaftlich anerkannte Position verschafft. Und nicht zuletzt hat der utopische Überschuß, der der Universitätsidee innewohnt, auch ein kritisches Potential bewahrt, das mit den zugleich universalistischen und individualistischen Grundüberzeugungen des okzidentalen Rationalismus in Einklang stand und von Zeit zu Zeit für eine Erneuerung der Institution wieder belebt werden konnte.

\section{Ideen zu einer Universitätsreform in den sechziger Jahren: JASPERS, SCHELSKY und die SDS-Hochschuldenkschrift}

Das jedenfalls glaubten die Reformer Anfang der sechziger Jahre. Nach 1945 hatte der erste Impuls zur Erneuerung nicht ausgereicht. Neben der materiellen Erschöpfung bestand eine Erschöpfung des korporativen Bewußtseins. Die Universitätsidee hatte in der Traditionsgestalt des Mandarinenbewußtseins auch die Nazis überlebt; aber durch erwiesene Ohnmacht gegen oder gar Komplizenschaft mit dem Naziregime war sie vor aller Augen ihrer Substanzlosigkeit überführt worden. Immerhin blieben nach 1945 die Traditionalisten der Humboldtschen Idee auch in der Defensive stark genug, um Reformversuche hinzuhalten und sich mit den Pragmatikern des Ende der fünfziger Jahre gegründeten Wissenschaftsrats zu arrangieren. Das unvermeidlich gewordene quantitative Wachstum der Universitäten vollzog sich dann als ein Ausbau in unveränderten Strukturen (s. ElLwEIN 1985, S. 238).

In dieser Situation greift JASPERS wiederum auf HUMBOLDT zurück; SCHELSKY und die Studenten des SDS versuchen eine kritische Aneignung desselben Erbes aus einer gewissen sozialwissenschaftlichen Distanz, indem sie ihren Reformvorschlägen eine nüchterne Diagnose des inzwischen eingetretenen Strukturwandels der Universität voranschicken. Im Hintergrund stehen schon die internationalen Vergleiche der Bildungssoziologen, die Bedarfsanalysen der Bildungsökonomen, die bürgerrechtlichen Postulate der Bildungspolitiker. Alles das faßt SCHELSKY unter dem Titel „Sachgesetzlichkeiten“ zusammen. Denn diese Prozesse haben einen systemischen Charakter und erzeugen Strukturen, die sich von der Lebenswelt ablösen; sie höhlen das korporative Bewußtsein der Universität aus, sie zersprengen jene Einheitsfiktionen, die Humboldt, SCHLEIERMACHER und ScHElling einst mit der totalisierenden Kraft der wissenschaftlichen Reflexion begründen wollten. Interessanterweise entscheidet sich SCHELSKY aber ebensowenig wie die linken Reformer für eine bloße Anpassung der Universitäten an die Sachgesetzlichkeiten; er setzt nicht auf die Art von technokratischer Dauerreform, die sich inzwischen tatsächlich eingespielt hatte. Diese Option hätte seine damals entwickelte Technokratietheorie sogar erwarten lassen. Stattdessen schöpft SCHELSKY aus dem Fundus der Humboldtschen Ideen, um dazu aufzurufen, die Sachgesetzlichkeiten „zu gestalten": „Das Entscheidende ist nun, daß diese sachgesetzlichen Entwicklungstendenzen einseitig sind ..., daß dazu eine Rückbindung und gestaltende Gegenkräfte ins Spiel treten müssen, die nicht selbstverständlich sind und nur in schöpferischer Anstrengung vollzogen werden können“ (SCHELSKY 1963, S. 275). Das 
ausdifferenzierte Wissenschaftssystem soll eben nicht nur mit Wirtschaft, Technik und Verwaltung zusammenwachsen, sondern über die traditionelle Bündelung ihrer Funktionen in der Lebenswelt verwurzelt bleiben. Und wiederum soll diese Funktionsbündelung aus der Struktur der Wissenschaft selbst erklärt werden.

Die theoretisch anspruchsvollen Reforminitiativen der frühen sechziger Jahre gehen also noch einmal von der Konzeption einer Wissenschaft aus, der man doch noch eine irgendwie einheitsstiftende Kraft zutrauen darf; und wiederum wird die Universität nur als deren äußere, organisatorische Gestalt begriffen. Natürlich hatte sich die Stellung der Philosophie zu den Wissenschaften inzwischen so verändert, daß nicht länger sie selbst das Zentrum der ausdifferenzierten Fachwissenschaften bildete. Aber wer sollte den Platz einnehmen? War es überhaupt nötig, an der Idee der Einheit der Wissenschaften festzuhalten? Die totalisierende Kraft des Wissenschaftsprozesses konnte gewiß nicht mehr als Synthese gedacht und durch einen metaphysischen Gegenstandsbezug zum Absoluten oder zur Welt im ganzen gesichert werden. Eine Theorie, die den Zugriff aufs Ganze - sei es direkt oder durch die Fachwissenschaften - riskiert hätte, stand nicht mehr zur Diskussion.

Eine vergleichsweise konventionelle Antwort gibt JASPERs. Er gesteht zu, daß die Rationalität der zieloffenen, allein methodisch bestimmten Erfahrungswissenschaften rein prozedural ist und eine inhaltliche Einheit im unvorhersehbar sich ausdifferenzierenden Fächerkanon nicht mehr begründen kann; aber der in die Peripherie zunächst abgedrängten, auf die Aufgaben der Existenzerhellung und der Analyse eines nicht objektivierbaren Umgreifenden zurückgenommenen Philosophie will JASPERS dann doch eine Sonderrolle gegenüber den freigelassenen Disziplinen vorbehalten. Die Wissenschaften sollen sogar der Führung durch die Philosophie bedürfen, weil nur diese das Motiv des unbedingten Wissenwollens und den Habitus der wissenschaftlichen Denkungsart durch Reflexion auf die Voraussetzungen und durch Vergewisserung der leitenden Ideen der Forschung sichern könne.

Weniger idealistisch sind SCHELSKYs Überlegungen, der die Philosophie durch eine Theorie der Wissenschaften ersetzt. Er geht von einer Dreiteilung des Fächerkanons in Natur-, Sozial- und Geisteswissenschaften aus. Die Fächer entfalten sich autonom; die drei Fachgruppen sind aber mit ihren spezifischen Wissensformen auf je andere Weise mit der modernen Gesellschaft funktional verzahnt. Sie können nicht mehr insgesamt durch philosophische Reflexion umgriffen werden; die Philosophie wandert vielmehr in die Wissenschaften ab und nistet sich in ihnen ein als eine Selbstreflexion der jeweiligen Disziplin. Für die fiktiv gewordenen Einheiten der Humboldtschen Universität entsteht so ein Äquivalent: „Indem die Philosopie aus den Fachwissenschaften hervorgeht und, diese zu ihrem Gegenstand machend, kritisch transzendiert, gewinnt sie indirekt wieder das Ganze der wissenschaftlichen Zivilisation als ihren Gegenstand. Indem sie die Grenzen der Bedingungen der Einzelwissenschaften erforscht, hält sie diese offen ... gegenüber der Verengung ihrer Weltbezüge" (SCHELSKY 1963, S. 290).

Ich selbst habe mich in der gleichen Zeit zum Anwalt einer materialen Wissenschaftskritik gemacht, welche die Verschränkung von methodischen Grundlagen, globalen Hintergrundannahmen und objektiven Verwertungszusammenhängen aufklären sollte (HABERMAS 1981, S. $101 \mathrm{ff}$. und 134ff.). Ich hatte die gleiche Hoffnung wie SCHELSKY, daß in dieser Dimension der wissenschaftskritischen 
Selbstreflexion die lebensweltlichen Bezüge der Forschungsprozesse aus diesen selbst heraus transparent gemacht werden könnten, und zwar nicht nur die Bezüge zu den Verwertungsprozessen wissenschaftlicher Informationen, sondern vor allem die Bezüge zur Kultur im ganzen, zu allgemeinen Sozialisationsvorgängen, zur Fortbildung von Traditionen, zur Aufklärung der politischen Öffentlichkeit.

Noch ein anderes Element des Humboldtschen Erbes lebte mit diesen Reforminitiativen wieder auf. Ich meine die exemplarische Bedeutung, die der Wissenschaftsautonomie über die grundrechtliche Garantie der Freiheit von Lehre und Forschung hinaus zugewiesen wurde. JASPERS verstand unter Wissenschaftsautonomie die Verwirklichung eines international verzweigten Kommunikationsnetzes, das den freien gegen den totalen Staat schützen würde (s. JASPERS 1961, S. $33 \mathrm{ff}$.). SCHELSKY verlieh dem eine personalistisch-existentielle Wendung: Wissenschaftsautonomie bedeutete die in pflichtgemäßer Einsamkeit eingeübte Distanzierung von, und die sittliche Souveränität gegenüber Handlungszwängen wie systemischen Verdinglichungen, die aus den gestaltungsbedürftigen Sachgesetzlichkeiten der modernen Gesellschaft resultierten ${ }^{7}$. Und für die Autoren der SDS-Hochschuldenkschrift, für die linken Reformer überhaupt verband sich mit dem, was wir damals als Demokratisierung der Hochschule verteidigt haben, zwar nicht die Übertragung von Modellen staatlicher Willensbildung auf die Universität, nicht die Bildung eines Staates im Staat, aber doch die Erwartung einer exemplarisch gemeinten politischen Handlungsfähigkeit in Form einer partizipatorischen Selbstverwaltung.

Jene Zielvorstellungen, die sich einer kritischen Aneignung der Universitätsidee verdankten, sind nicht realisiert worden. In einem Nachtrag zu seinem Buch erklärt SCHELSKY 1970 das Scheitern der Reformen damit, daß sich das Wissenschaftssystem unter dem Zwang zur Komplexitätssteigerung hochgradig ausdifferenziert hat und daher in seinen verschiedenen Funktionen „nicht mehr von einem gemeinsamen Leitbild her zusammengehalten werden könne" (SCHELSKY ${ }^{2} 1970$, S. 243). Der verräterische Ausdruck „Leitbild“ verweist auf Prämissen, die vielleicht wirklich zu naiv waren, um mit der Differenzierungsdynamik der Forschung selbst Schritt zu halten. Unrealistisch war offenbar die Annahme, daß sich dem disziplinär organisierten Forschungsbetrieb eine Reflexionsform einpflanzen ließe, die nicht aus der Logik der Forschung selbst hervorgetrieben wird. Die Geschichte der modernen Erfahrungswissenschaften lehrt, daß „normal science" durch Routinen gekennzeichnet ist und durch einen Objektivismus, der den Forschungsalltag gegen Problematisierungen abschirmt. Reflexionsschübe werden durch Krisen ausgelöst, aber auch dann vollzieht sich die Verdrängung degenerierender durch neue Paradigmen eher naturwüchsig. Wo hingegen Grundlagenreflexion und Wissenschaftskritik auf Dauer gestellt werden, etablieren sie sich - wie die Philosophie selbst - als Fach neben Fächern. Nicht weniger unrealistisch war die Erwartung, daß sich die kollegiale Selbstverwaltung der Hochschulen allein durch eine funktional gegliederte Partizipation der beteiligten Gruppen mit politischem Leben erfüllen würde - erst recht, wenn die Reform gegen den Willen der Professoren auf dem Verwaltungswege erzwungen werden mußte.

Wenn aber der innere Zusammenhang der Universität nicht einmal mehr unter diesen Prämissen zu retten war, müssen wir uns dann nicht doch eingestehen, daß diese Institution auch ganz gut ohne jene liebgewordene Idee auskommt, die sie einmal von sich selbst gehabt hat? 


\section{Worin könnte ein integrierendes Selbstverständnis der Universität heute gegründet sein?}

Die sozialwissenschaftliche Systemtheorie trifft mit der Wahl ihrer Grundbegriffe eine Vor-entscheidung: sie unterstellt, daß alle sozialen Handlungsbereiche unterhalb der Ebene normativer Orientierungen durch wertneutrale Steuerungsmechanismen wie Geld oder administrative Macht zusammengehalten werden. Für die Systemtheorie gehört die integrative Kraft von Ideen und Institutionen apriori zum mehr oder weniger funktionalen Überbau eines Substrats von Handlungs- und Kommunikationsflüssen, die systemisch aufeinander abgestimmt sind und dazu keiner Normen bedürfen. In modernen Gesellschaften bildeten sich autonome, keineswegs miteinander verschränkte Subsysteme heraus, die auf genau eine Funktion, auf nur eine Art von Leistungen spezialisiert seien.

Diese Behauptung zieht ihre Evidenz aus dem Anblick einer über Geld gesteuerten Wirtschaft oder einer über Machtbeziehungen regulierten staatlichen Verwaltung. Problematisch ist dabei die Verallgemeinerung dieser Beobachtung auf alle Handlungssysteme - und erst daraus bezieht die Systemtheorie ihre Pointe. Sie suggeriert, $\mathrm{da} ß$ jeder Handlungsbereich, wenn er nur au courant bleiben will mit der gesellschaftlichen Modernisierung, diese Gestalt funktional spezifizierter, über Steuerungsmedien ausdifferenzierter, voneinander entkoppelter Teilsysteme annehmen müsse. Sie fragt gar nicht erst, ob das für alle Handlungsbereiche gelten kann, beispielsweise für kulturelle Handlungssysteme wie den Wissenschaftsbetrieb, dessen Kernsektor bisher immer noch in einem funktionsbündelnden Institutionensystem untergebracht ist - in wissenschaftlichen Hochschulen, die keineswegs in gleicher Weise wie kapitalistische Unternehmungen oder internationale Behörden dem Horizont der Lebenswelt entwachsen sind. Es muß sich erst noch zeigen, ob sich die aus der Universität ausgelagerte Groß- und Grundlagenforschung vom generativen Prozeß der in den Hochschulen organisierten Wissenschaft ganz wird lösen können - ob sie ganz auf eigenen Beinen wird stehen können oder doch parasitär bleibt. $\mathrm{Da}$ eine von universitären Formen, also auch von der Forschung abgeschnittene wissenschaftliche Fachausbildung Schaden nehmen müßte, ist mindestens eine plausible Vermutung. Gegen die systemtheoretische Überverallgemeinerung spricht vorerst die Erfahrung, die SCHELSKY so formuliert: „Das Einmalige in der institutionengeschichtlichen Entwicklung der modernen Universität besteht darin, daß sich in diesem Falle die Funktionsdifferenzierung innerhalb der gleichen Institution vollzieht und kaum ein Funktionsverlust durch Abgabe von Aufgaben an andere Organisationen eintritt. Man kann im Gegenteil eher von einer Funktionsbereicherung, mindestens von einem Bedeutungsgewinn und einer Verbreiterung der Funktionsbereiche der Universität in ihrer Entwicklung während des letzten Jahrhunderts sprechen" (1961, S. 267).

So geht denn auch TAlCOTT PARsons in seinem für die Hochschulsoziologie bis heute maßgebenden Buch über die Amerikanische Universität (s. PARsons/PlatT 1973, S. $90 \mathrm{ff}$.) unbefangen davon aus, daß das Hochschulsystem vier Funktionen gleichzeitig erfüllt: die Kernfunktion (a) der Forschung und der Förderung des wissenschaftlichen Nachwuchses geht Hand in Hand mit (b) der akademischen Berufsvorbereitung (und der Erzeugung technisch verwertbaren Wissens) auf der einen Seite, mit (c) Aufgaben der allgemeinen Bildung und (d) Beiträgen zu 
kultureller Selbstverständigung und intellektueller Aufklärung andererseits. PARsONS kann sich auf das institutionell stärker differenzierte Hochschulsystem in den USA beziehen und die ersten drei der genannten Funktionen verschiedenen Institutionen - den graduate schools, den professional schools und den colleges zuordnen. Aber jede dieser Institutionen ist in sich noch einmal so differenziert, daß sie sich jeweils mit verschiedener Gewichtung nach allen Funktionsbereichen hin verzweigt. Nur die vierte Funktion hat keine eigene Trägerinstitution; sie wird über die Intellektuellenrolle der Professoren erfüllt. Wenn man bedenkt, daß PARsons in dieser vierten Funktion beides unterbringt: nicht nur die nach außen gerichteten, an die Öffentlichkeit adressierten Aufklärungsleistungen, sondern auch die Reflexion auf die eigene Rolle der Wissenschaften und auf das Verhältnis der kulturellen Wertsphären Wissenschaft, Moral und Kunst zueinander, erkennt man, daß dieser Funktionenkatalog in verwandelter Gestalt genau das wiedergibt, was die preußischen Reformer einst als „Einheiten“ fingiert hatten: als Einheit von Forschung und Lehre, als Einheit von Wissenschaft und allgemeiner Bildung, als Einheit von Wissenschaft und Aufklärung und als Einheit der Wissenschaften.

Diese letzte Idee hat freilich ihre Bedeutung gravierend verändert; denn die offen ausdifferenzierte Mannigfaltigkeit der wissenschaftlichen Disziplinen stellt nicht mehr als solche das Medium dar, das alle jene Funktionen bündeln kann. Nach wie vor stehen jedoch die universitären Lernprozesse nicht nur im Austausch mit Wirtschaft und Verwaltung, sondern in einem inneren Zusammenhang mit den Reproduktionsfunktionen der Lebenswelt. Hinausgehend über akademische Berufsvorbereitung leisten sie mit der Einübung in die wissenschaftliche Denkungsart, d.h. in eine hypothetische Einstellung gegenüber Tatsachen und Normen, ihren Beitrag zu allgemeinen Sozialisationsvorgängen; hinausgehend über Expertenwissen leisten sie mit fachlich informierten zeitdiagnostischen Deutungen und sachbezogenen politischen Stellungnahmen einen Beitrag zur intellektuellen Aufklärung; hinausgehend über Methoden- und Grundlagenreflexion leisten sie mit den Geisteswissenschaften auch eine hermeneutische Fortbildung von Traditionen, mit Theorien der Wissenschaft, der Moral, der Kunst und Literatur einen Beitrag zur Selbstverständigung der Wissenschaften im Ganzen der Kultur. Es ist die universitäre Form der Organisation wissenschaftlicher Lernprozesse, die auch noch die ausdifferenzierten Fachdisziplinen über die gleichzeitige Erfüllung dieser verschiedenen Funktionen in der Lebenswelt verwurzelt.

Die Ausdifferenzierung der Fächer verlangt freilich eine entsprechend starke Differenzierung im Innern der Universität. Verschiedene Funktionen werden von verschiedenen Personengruppen an verschiedenen institutionellen Orten mit verschiedener Gewichtung wahrgenommen. Das korporative Bewußtsein verdünnt sich mithin zu dem intersubjektiv geteilten Wissen, daß zwar andere anderes tun als andere, daß aber alle zusammengenommen, indem sie auf diese oder jene Art Wissenschaft treiben, nicht nur eine, sondern ein Bündel von Funktionen erfüllen. Diese bleiben über den arbeitsteilig betriebenen Wissenschaftsprozeß $B$ miteinander verschränkt. Daß die Funktionen gebündelt bleiben, läßt sich aber heute kaum noch, wie SCHELSKY meinte, auf die Bindungskraft des normativen Leitbildes der deutschen Universität zurückführen. Wäre das überhaupt wünschenswert? Um das korporative Selbstverständnis der Universität wäre es schlecht bestellt, wenn es in so etwas wie einem normativen Leitbild verankert wäre; denn Ideen kommen und 
gehen. Der Witz der alten Universitätsidee bestand gerade darin, daß sie in etwas Stabilerem gegründet werden sollte - eben in dem auf Dauer ausdifferenzierten Wissenschaftsprozeß selber. Wenn nun aber die Wissenschaft als ein solcher Ideenanker nicht mehr taugt, weil die Mannigfaltigkeit der Disziplinen keinen Raum mehr läßt für die totalisierende Kraft sei es einer alles umfassenden philosophischen Grundwissenschaft oder auch nur einer aus den Fächern selbst hervorgehenden Reflexionsform materialer Wissenschaftskritik, worin könnte dann ein integrierendes Selbstverständnis der Korporation gegründet sein?

Die Antwort findet sich bereits bei SCHLEIERMACHER: „Das erste Gesetz jedes auf Erkenntnis gerichteten Bestrebens (ist): Mitteilung; und in der Unmöglichkeit, irgendetwas auch nur für sich allein ohne Sprache hervorzubringen, hat die Natur selbst dieses Gesetz ganz deutlich ausgesprochen. Daher müssen sich rein aus dem Triebe nach Erkenntnis ... auch alle zu seiner zweckmäßigen Befriedigung nötigen Verbindungen, die verschiedenen Arten der Mitteilung und der Gemeinschaft aller Beschäftigungen von selbst gestalten." Ich stütze mich auf diesen einen von SCHLEIERMACHERS „Gelegentlichen Gedanken über Universitäten im deutschen Sinne“ (in: ANRICH 1959, S. 224) ohne Sentimentalität, weil ich im Ernst meine, daß es die kommunikativen Formen der wissenschaftlichen Argumentation sind, wodurch die universitären Lernprozesse in ihren verschiedenen Funktionen letzlich zusammengehalten werden. SCHLEIERMACHER hält es für „einen leeren Schein, als ob irgendein wissenschaftlicher Mensch abgeschlossen für sich in einsamen Arbeiten und Unternehmungen lebe"; so sehr er in der Bibliothek, am Schreibtisch, im Laboratorium alleine zu arbeiten scheint, so unvermeidlich sind seine Lernprozesse eingelassen in eine öffentliche Kommunikationsgemeinschaft der Forscher. Weil das Unternehmen kooperativer Wahrheitssuche auf diese Strukturen einer öffentlichen Argumentation verweist, kann Wahrheit - oder sei's auch nur die in der community of investigators erworbene Reputation - niemals zum bloßen Steuerungsmedium eines selbstgeregelten Subsystems werden. Die wissenschaftlichen Disziplinen haben sich in fachinternen Öffentlichkeiten konstituiert, und nur in diesen Strukturen können sie sich ihre Vitalität erhalten. Die fachinternen Öffentlichkeiten schießen zusammen und verzweigen sich wiederum in den universitätsöffentlichen Veranstaltungen. Der altväterliche Titel des Ordentlichen öffentlichen Professors erinnert an den Öffentlichkeitscharakter der Vorlesungen, der Seminare und der wissenschaftlichen Kooperation in den Arbeitsgruppen der angegliederten Institute. Es gilt eben nicht nur für die Idealform des Seminars, sondern für die Normalform der wissenschaftlichen Arbeit, was HuMBoLDT vom kommunikativen Umgang der Professoren mit ihren Studenten gesagt hat: der Lehrer würde, ,wenn sie (Studenten und jüngere Kollegen) sich nicht von selbst um ihn versammelten, sie aufsuchen, um seinem Ziele näher zu kommen durch die Verbindung der geübten, aber eben darum auch leichter einseitigen und schon weniger lebhaften Kraft mit der schwächeren und noch parteiloser nach allen Richtungen mutig hinstrebenden" (in ANRICH 1959, S. 378). - Dieser Satz bewahrheitet sich in dem fester organisierten Betrieb eines Max-Planck-Institutes nicht weniger als in einem philosophischen Seminar. Noch jenseits der Universität behalten wissenschaftliche Lernprozesse etwas von ihrer universitären Ursprungsform. Sie alle leben von der Anregungs- und Produktivkraft eines diskursiven Streites, der die promissory note des überraschen- 
den Argumentes mit sich führt. Die Türen stehen offen, in jedem Augenblick kann ein neues Gesicht auftauchen, ein neuer Gedanke unerwartet eintreten.

Ich möchte nun nicht den Fehler wiederholen, die Kommunikationsgemeinschaft der Forscher ins Exemplarische zu stilisieren. Im egalitären und universalistischen Gehalt ihrer Argumentationsformen drücken sich zunächst nur die Normen des Wissenschaftsbetriebs aus, nicht die der Gesellschaft im ganzen. Aber sie haben auf prononcierte Weise Teil an jener kommunikativen Rationalität, in deren Formen moderne, also nicht festgestellte, leitbildlose Gesellschaften sich über sich selbst verständigen müssen.

\section{Anmerkungen}

* Vortrag, gehalten auf Einladung des Stadttheaters Heidelberg aus Anlaß der 600-JahrFeier der Universität.

1 Nicht berücksichtigt sind weitere 94 Fachhochschulen und 26 Kunsthochschulen. Vgl. KOHLER, H./NAuMANN, J.: Trends der Hochschulentwicklung 1970 bis 2000. In: Recht der Jugend und des Bildungswesens 32 (1984), H. 6, S. $419 \mathrm{ff}$. Ein Überblick in: MAX-PLANCKInSTITUT FUR BILDUNGSFORSCHUNG (1984), S. $228 \mathrm{ff}$.

2 Dafür sprechen auch die nationalen Ungleichzeitigkeiten der Karrieren von bildungsreformerischen Vorschlägen. So haben beispielsweise im vergangenen Jahr die 50 Professoren am Collège de France dem französischen Präsidenten Bildungsreformempfehlungen vorgelegt, die in Tenor und Zielsetzung an das Reformklima der späten sechziger Jahre in der Bundesrepublik erinnern. Die von Pierre Bordieu inspirierten Empfehlungen sind erschienen in: Neue Sammlung 25 (1985), H. 3.

3 „Wenigstens ein anständiges und edeles Leben gibt es für den Staat ebensowenig als für den einzelnen, ohne mit der immer beschränkten Fertigkeit auf dem Gebiete des Wissens doch einen allgemeinen Sinn zu verbinden. Für alle diese Kenntnisse macht der Staat natürlich und notwendig eben die Voraussetzung wie der einzelne, $\mathrm{da} ß$ sie in der Wissenschaft müssen begründet sein, und nur durch sie recht können fortgepflanzt und vervollkommnet werden" (SCHLEIERMACHER, F. [1808] 1959, S. 226).

$4 \mathrm{Zu}$ den Reaktionen der deutschen Philosophie auf diese neue Situation vgl. ScHNÄDELBACH, H. (1983), S. $118 \mathrm{ff}$.

5 Vgl. meine Rezension des Buches von Ringer: Die deutschen Mandarine, in: Habermas, J. (1981), S. $485 \mathrm{ff}$.

6 Vgl. zu dieser These J. Klüwer (1983).

7 Schelsky (1963, S. 299): „Die Gefahr, daß der Mensch sich nur in äußere, umweltverändernde Handlung auslegt und alles, den anderen Menschen und sich selbst, in dieser Gegenstandsebene der konstruktiven Handlung festhält und behandelt. Diese neue Selbstentfremdung des Menschen, die ihm die innere Identität seiner selbst und des anderen rauben kann, diese neue metaphysische Versuchung des Menschen, ist die Gefahr, $\mathrm{da} ß$ der Schöpfer sich in sein Werk, der Konstrukteur in seine Konstruktion verliert. Der Mensch schaudert zwar davor zurück, sich restlos in die selbstproduzierte Objektivität, in ein konstruiertes Sein, zu transferieren und arbeitet doch unaufhörlich am Fortgang dieses Prozesses der wissenschaftlich-technischen Selbstobjektivierung. “

\section{Literatur}

ANrich, E. (Hrsg.): Die Idee der Deutschen Universität. Darmstadt 1959.

EllweIN, T.: Die deutsche Universität. Königstein 1985. 
Fichte, J.G.: Deduzierter Plan einer in Berlin zu errichtenden höheren Lehranstalt. In: ANRICH (1959).

Friedeburg, L. von: Elite - elitär? In: Becker, G. u. a. (Hrsg.): Ordnung und Unordnung. Weinheim 1986.

Habermas, J.: Vom sozialen Wandel akademischer Bildung. In: Ders.: Kleine Politische Schriften I-IV. Frankfurt a. M. S. $101 \mathrm{ff}$.

Habermas, J.: Universität in der Demokratie-Demokratisierung der Universität. In: Ders.: Kleine Politische Schriften I-IV. Frankfurt a. M. 1981, S. $134 \mathrm{ff}$.

Habermas, J.: Philosophisch-Politische Profile. Frankfurt a.M. 1981.

HüFner, K./NaumanN, J.: Konjunkturen der Bildungspolitik in der BRD. Stuttgart 1977.

Húfner, K./NaumanN, J./Köhler, H./Pfeffer, G.: Hochkonjunktur und Flaute: Bildungspolitik in der BRD. Stuttgart 1986.

HUMBOLDT, W. vON: Über die innere und äußere Organisation der Höheren wissenschaftlichen Anstalten (1810). In: ANRICH, E. (Hrsg.) (1959).

JASPERS, K./Rossmann, K.: Die Idee der Universität. Heidelberg 1961.

KLUWWER, J.: Universität und Wissenschaftssystem. Frankfurt a. M. 1983.

Martens, E./Schnädelbach, H.: Philosophie - Grundkurs. Hamburg 1985.

Max-Planck-Institut fur Bildungsforschung: Das Bildungswesen in der Bundesrepublik. Hamburg 1984.

Nitsch, W./Gerhardt, U./OfFe, C./Preuss, U.K.: Hochschule in der Demokratie. Neuwied 1965.

Parsons, T./Platt, G.M.: The American University. Cambridge, Mass. 1973.

Reumann, K.: Verdunkelte Wahrheit. In: FAZ v. 24. März 1986.

Ringer, F.K.: The Decline of the German Mandarins. Cambridge, Mass. 1969.

Schelling, F. W. J.: Vorlesungen über die Methode des akademischen Studiums (1802). In: ANRICH, E. (Hrsg.) (1959).

SCHELSKY, H.: Einsamkeit und Freiheit. Hamburg 1963. ${ }^{2} 1970$.

SCHLEIERMACHER, F.: Gelegentliche Gedanken über Universitäten im deutschen Sinn [1808]. In: ANRICH (1959).

Schnadelbach, H.: Philosophie in Deutschland 1931-1933. Frankfurt a.M. 1983.

WISSENSCHAFTSRAT: Empfehlungen und Stellungnahmen. Köln 1984.

WISSENSCHAFTSRAT: Empfehlungen zum Wettbewerb im deutschen Hochschulsystem. Köln 1985. (a)

WisSENSCHAFTSRAT: Empfehlungen zur klinischen Forschung. Köln 1985. (b)

WISSENSCHAFTSRAT: Empfehlungen zur Struktur des Studiums. Köln 1986.

\section{Abstract}

\section{The Concept of University - Learning Processes}

The article starts with a characterization of the concept of University as formulated by the Prussian reformers of higher education in the early 19th century. Subsequently, major reform treatises of the sixties - JASPERS, SCHELSKY, and the SDS memorandum - are analyzed with regard to their respective links to that tradition and their reformulations of the classical concept of University. As a major issue emerges the question whether institutions of higher learning with their bundle of functions continue to need an integrating self-concept or can quite easily do without it - the University just being a part of the larger differentiated science system.

Anschrift des Autors:

Prof. Dr. Jürgen Habermas, Johann Wolfgang von Goethe Universität, Fachbereich Philosophie, Dantestr. 4-6, 6000 Frankfurt 11 